ARTíCULOS DE INVESTIGACIÓN

\title{
Educación legal clínica y servicios legales gratuitos a migrantes y refugiados en Colombia: Relato de una experiencia
}

\author{
Educação legal clínica e serviços jurídicos gratuitos para migrantes \\ e refugiados na Colômbia: Relato de uma experiência \\ Clinical legal education and free legal services for migrants and refugees \\ in Colombia: An experience tale
}

\author{
Gracy Pelacani (iD y Carolina Moreno \\ Universidad de los Andes, Colombia
}

\begin{abstract}
RESUMEN El presente trabajo reflexiona, a partir de una experiencia concreta, sobre los aportes de la educación legal clínica para lograr un mayor acceso a la justicia y garantía de los derechos de la población migrante y refugiada en Colombia. Tras presentar un breve contexto, el texto ahonda en la forma como, desde el Consultorio Jurídico de la Universidad de los Andes (Bogotá, Colombia) y luego, desde la Clínica Jurídica para Migrantes de la misma universidad, se ha respondido a la creciente necesidad de servicios legales gratuitos y especializados por parte de esta población. El artículo reflexiona sobre los desafíos específicos de abogar por los derechos de las personas migrantes y refugiadas en el contexto colombiano. En particular y a partir de algunos casos acompañados por los estudiantes de la Clínica, el trabajo profundiza sobre los aprendizajes adquiridos y sobre los desafíos encontrados. El artículo concluye afirmando que, en este contexto, la educación legal clínica muestra su potencial pedagógico y social transformador, al tiempo que representa una ocasión de reflexión crítica sobre los límites del derecho y los conflictos éticos que subyacen a la profesión legal.
\end{abstract}

PALABRAS CLAVE Educación legal clínica, educación experiencial, cambio social, migración, Colombia.

RESUMO Este trabalho reflete sobre as contribuições da educação legal clínica pra conseguir um maior aceso a justiça e garantir os direitos das pessoas migrantes e refugiadas na Colômbia, a partir de uma experiência específica. Com este objetivo, após apresen- 
tar um breve contexto, o texto investiga a maneira como o Consultorio Jurídico de la Universidad de los Andes (Bogotá, Colômbia) y, posteriormente, a Clínica Jurídica para Migrantes da mesma universidade, responderam as necessidades cada vez maiores de serviços jurídicos gratuitos e especializados por parte dessa população. Assim, o artigo reflete sobre os desafios específicos envolvidos em defender os direitos das pessoas migrantes e refugiadas no contexto colombiano. Em particular, a partir de alguns casos acompanhados pelos estudantes da Clínica, este trabalho aprofunda sobre as aprendizagens e os desafios encontrados. Este artigo se conclui afirmando que, neste contexto, a educação legal clínica mostra o seu potencial pedagógico e social transformador e, ao mesmo tempo, representa uma ocasião para refletir criticamente sobre os limites do direto e os conflitos éticos da profissão legal.

PALAVRAS-CHAVE Educação legal clínica, educação experiencial, mudança social, migração, Colômbia

ABSTRACT The essay reflects on the contributions of Clinical Legal Education to achieve greater access to justice and rights for migrants and refugees in Colombia, based on a specific experience. With this objective in mind, after providing a brief context, the text investigates how the Consultorio Jurídico de la Universidad de los Andes (Bogota, Colombia), and, later, the Legal Clinic for Migrants at the same university, responded to the increasing needs for specialized and free legal services by this population. Thus, the article reflects on the specific challenges involved in advocating for migrants and refugees' rights in the Colombian context. In particular, based on some cases accompanied by the students of the Clinic, this work deepens on the lessons learned and challenges encountered. The article concludes by stating that, in this context, Clinical Legal Education shows its pedagogical and social transformative potential, and, at the same time, it is an occasion to critically reflect on the limits of the law and on the ethical conflicts that underlie the legal profession.

KEYWORDS Clinical Legal Education, experiential education, social change, migration, Colombia

\section{Introducción}

Antes de que la pandemia por covid-19 nos obligara a reducir drásticamente nuestros contactos con la población migrante y refugiada, pasamos una tarde con nuestros estudiantes atendiendo a estas personas. Fuera de una sala con espacio para unas pocas mesas y sillas, la fila no dejaba de aumentar y con mucho esfuerzo comunicamos a quienes seguían llegando que ese día no íbamos a poder atenderlos. "Volveremos», les dijimos, sin saber que la pandemia nos iba a impedir cumplir con este propósito por mucho tiempo.

Esta historia ilustra una experiencia de educación legal clínica y las oportuni- 
dades que ofrece a los estudiantes de experimentar de primera mano la profesión legal, sus conflictos éticos y los retos que implica atender las necesidades legales de una población en situación de vulnerabilidad, condición en la que se encuentran las personas migrantes y refugiadas que acuden a la Clínica Jurídica para Migrantes de la Universidad de los Andes.

De los más de 5,9 millones de nacionales venezolanos que se estima han dejado su país de origen, alrededor de 1 millón 700 mil se encuentran en territorio colombiano, siendo Colombia el país de destino del más alto número de estas personas en la región latinoamericana y a nivel global (ACNUR y OIM, 2021). La gestión de esta migración ha sido compleja, considerando que la mayoría de la población venezolana en Colombia se encuentra en situación migratoria irregular (Migración Colombia, 2021), lo que constituye el primer obstáculo para la protección y garantía de sus derechos.

En general, Colombia cuenta con una normatividad migratoria compuesta por actos administrativos, que ha venido desarrollándose de forma reactiva y fragmentaria sin que mediara un enfoque de largo plazo y de derechos (Moreno y Pelacani, 2021: 184-188; Pelacani y otros, 2021). A esta situación ha contribuido que, solo desde agosto del 2021, Colombia cuenta con una ley migratoria, aunque esta hace poco más que confirmar el statu quo y, sobre todo, carece de un enfoque de derechos ${ }^{1}$ (Pelacani, 2020).

En este escenario, la Corte Constitucional colombiana ha jugado un rol esencial contribuyendo de forma fundamental, en algunos casos, a la protección de los derechos de la población migrante y refugiada (Moreno y Pelacani, 2020: 146-152). Por otro lado, en muchos casos los jueces de tutela muestran un conocimiento limitado en materia migratoria y, en particular, frente a la migración proveniente de Venezuela, lo que se refleja en ocasiones con la adopción de órdenes de imposible cumplimiento.

Ante estos desafíos, en este texto reflexionamos sobre cómo - desde la educación legal clínica-es posible contribuir a un mayor acceso a la justicia y a derechos de la población migrante y refugiada en Colombia. En especial, nos enfocamos en la experiencia de la Clínica Jurídica para Migrantes de la Universidad de los Andes (Bogotá, Colombia), creada en junio de 2019, con el fin de atender la demanda creciente de servicios legales gratuitos por parte de esta población. Para ello, en la segunda sección de este escrito nos detenemos en la forma en que el Consultorio Jurídico de la Universidad de los Andes respondió ante las necesidades de la población migrante y refugiada, hasta llegar a la creación de la Clínica Jurídica para Migrantes. En la tercera parte, reflexionamos sobre las características de la atención legal a la población migrante y refugiada y los retos particulares que esta conlleva en Colombia. En el cuarto acápite, a través de algunos casos significativos adelantados

1. Véase Carolina Moreno, «La ley migratoria: Una necesidad imperiosa que un proyecto no satisface», Ámbito Jurídico, 17 de julio de 2020, disponible en https://bit.ly/3xi7 $\mathrm{O}_{4} \mathrm{~N}$. 
por la Clínica, mostramos las dificultades encontradas en la defensa de los derechos de esta población. Finalmente, concluimos afirmando que estos casos permitieron a los estudiantes experimentar de primera mano los límites del derecho y su injusticia, pero también su capacidad de transformar el entorno y ser un medio para avanzar los derechos de la población migrante y refugiada en Colombia. Así mismo, ha sido una oportunidad para reflexionar críticamente sobre su práctica jurídica y los conflictos éticos que la profesión legal implica.

\section{Respuesta frente a las necesidades legales de la población migrante y refugiada en Colombia}

\section{Las primeras iniciativas desde el Consultorio Jurídico de la Universidad de los Andes}

El consultorio jurídico es la práctica obligatoria que deben cumplir todos los estudiantes de derecho en Colombia para optar por el título profesional de abogados (Moreno y Hoyos Ceballos, 2021; Recalde, Luna Blanco y Bonilla Maldonado, 2017). Esta práctica se lleva a cabo al final de la carrera y de acuerdo con la estructura que cada facultad decida al interior de las instituciones de educación superior. En el desarrollo de esta práctica los estudiantes experimentan de primera mano la profesión legal, toda vez que la normativa colombiana los habilita para representar directamente los intereses de personas, sin la intermediación de un profesional del derecho, de acuerdo con la Ley 2.113 de 2021. Los servicios legales que ofrecen los consultorios jurídicos suplen las necesidades legales insatisfechas de personas que no pueden asumir el pago de abogados profesionales y demás costos asociados al acceso a la justicia.

Desde su creación en 1971, el Consultorio Jurídico de la Facultad de Derecho de la Universidad de los Andes, ubicado en Bogotá, Colombia, ofrece de forma ininterrumpida una práctica jurídica con distintas opciones para los estudiantes, pero siempre con un propósito doble: uno de carácter formativo y otro de responsabilidad social universitaria (García Suárez, 2019: 88-100). Por una parte, los estudiantes continúan con su proceso formativo como abogados y abogadas desde un enfoque basado en la experiencia. Por otra, la práctica posibilita el desarrollo de una labor social mediante la cual se ofrecen servicios legales gratuitos a personas en situación de vulnerabilidad.

A partir del año 2018 y en respuesta al fenómeno migratorio colombiano, el Consultorio puso en marcha sus primeras iniciativas de servicios legales gratuitos enfocados en las necesidades de la población migrante y refugiada en situación de pobreza. La primera estrategia fue la realización de la llamada «brigada temática», en la que, durante una semana, el Consultorio concentró su oferta de servicios legales en la población migrante y refugiada. Esta brigada, además de facilitar el acceso a los 
servicios del Consultorio, permitió que este espacio fuera conocido por esta población como uno seguro y disponible al que podían acudir para tramitar sus asuntos. Aunque esta iniciativa no estaba circunscrita a los nacionales venezolanos, lo cierto es que por las dimensiones y la composición de la población migrante y refugiada presente en Colombia, todas las personas atendidas en aquella oportunidad fueron ciudadanos venezolanos. De acuerdo con los datos recogidos por la coordinación administrativa del Consultorio, esta brigada jurídica contó con la participación de 39 estudiantes practicantes de derecho, quienes atendieron a un total de 160 personas migrantes venezolanas, durante 36 horas de trabajo.

Con posterioridad a la realización de la brigada jurídica se inició un incremento paulatino de la demanda de los servicios legales del Consultorio. De acuerdo con los datos arrojados por la plataforma CJDigital, que recoge la información sobre los servicios legales prestados en el Consultorio, en 2019 se recibieron ocho solicitudes de atención legal de personas migrantes y refugiadas. Para el año 2020 estas consultas ascendieron a 110 y a junio de 2021 se habían atendido 37 consultas relacionadas con la migración. El aumento en los casos sobre necesidades legales de personas migrantes y refugiadas posibilitó que los estudiantes del Consultorio explorarán la atención de casos sobre desconocimiento tanto de los derechos laborales de esta población como de su acceso a los servicios de salud.

El incremento progresivo de los casos de personas migrantes y refugiadas en situación de vulnerabilidad nos llevó a reflexionar sobre la capacidad del Consultorio para responder a estas necesidades. Fue entonces cuando comprendimos que el fenómeno migratorio en Colombia era estructural, y en tal sentido, requería el diseño y la implementación de estrategias legales más allá del litigio individual, que era la forma como entonces el Consultorio respondía a las cuestiones legales de estos grupos. Producto de esta reflexión, en junio de 2019 nació la Clínica Jurídica para Migrantes de la Facultad de Derecho de la Universidad de los Andes. En la siguiente sección describimos el tipo de labor que desde ahí se realiza, considerando los aprendizajes adquiridos desde su nacimiento, así como los desafíos que impone el trabajo clínico enfocado en población migrante y refugiada en Colombia.

\section{La Clínica Jurídica para Migrantes y la educación legal clínica}

Según se describió, la Clínica Jurídica para Migrantes es una de las opciones posibles para que los estudiantes del Consultorio puedan realizar su práctica jurídica. Por las características de la población que atiende y por sus necesidades, la Clínica trabaja simultáneamente en litigio individual así como estratégico y está organizada como un curso de la carrera. En este sentido, no solo es una oportunidad para que los estudiantes ofrezcan servicios legales a una población en situación de vulnerabilidad, sino también para adquirir y afianzar su conocimiento legal en derecho migratorio, 
una temática que no se enseña en la carrera de derecho en Colombia. Además, la formación se enmarca en la metodología de enseñanza clínica, cuyo eje central es la experiencia, esto es, el «hacer» de los estudiantes como abogados y abogadas.

En tal sentido, los estudiantes de la Clínica prestan asesoría legal gratuita a la población migrante y refugiada; representan sus intereses ante instancias administrativas y judiciales; realizan Talleres de Empoderamiento Legal (TEL) e instalan brigadas jurídicas en los sitios donde se encuentra la población en situación de pobreza. El trabajo que llevan a cabo las y los estudiantes cuenta con la orientación y el seguimiento permanentes de un equipo de profesoras de la facultad quienes, mediante encuentros plenarios e individuales con los estudiantes, acompañan el estudio de los casos, la definición de las estrategias legales a seguir y la gestión como tal de los asuntos. Así mismo, las profesoras definen las herramientas teóricas, normativas y prácticas que las y los practicantes requieren para su experiencia de aprendizaje en la Clínica.

De acuerdo con la plataforma CJDigital, los 286 casos atendidos por la Clínica desde su creación muestran que los asuntos que adelantan los estudiantes de la Clínica tienen que ver, principalmente, con el acceso al derecho a la salud (95 casos), solicitudes de reconocimiento de la condición de refugiado (88 casos), regularización migratoria (38 casos), nacionalidad (34 casos) y educación (16 casos). Ánfora, otra plataforma que permite caracterizar a los usuarios que se atienden en la Clínica, muestra que el 59\% de las personas que acudieron a los servicios de la Clínica se encontraban en situación migratoria irregular. Además, la Clínica ha atendido casos relevantes sobre expulsiones de personas migrantes y refugiadas, en las cuales se ha evidenciado la violación del derecho al debido proceso. ${ }^{2}$ También, las prolongadas cuarentenas que trajo la pandemia por covid-19 implicaron el surgimiento de una necesidad antes inexistente: desalojos de personas migrantes y refugiadas, quienes no pudieron pagar los arrendamientos de sus sitios de alojamiento (Proyecto Migración Venezuela, 2020).

El adelantamiento de estos casos, a partir del litigio individual, constituye un insumo fundamental para el diseño de estrategias de litigio estructural o de alto impacto, basado en el derecho de interés público (Courtis, 2007; Bonilla, 2018) y para las actividades de la Clínica enmarcadas en la educación legal clínica (ELC) (Bloch, 2010). Por su lado, el Litigio de Interés Público (LIP) promueve la participación de diversos actores, como universidades y organizaciones de la sociedad civil, en distintas acciones y actividades para consolidar la democracia y avanzar en la garantía de los derechos. En este sentido, actores usualmente excluidos de los escenarios más

2. Véase Catalina Lobo-Guerrero, «Así terminó la expulsión de 59 venezolanos durante las protestas del 2019", La Silla Vacía, 17 de noviembre de 2021, disponible en https://bit.ly/3cHUBJJ. Y de la misma autora, «Prohibido volver: El triste destino de Miguel Calderón», La Silla Vacía, 14 de noviembre de 2021, disponible en https://bit.ly/3dooHFH. 
tradicionales donde se define el marco normativo y la política pública, encuentran a través del LIP un medio y espacio para incidir y avanzar en sus intereses y agendas (González Morales, 2004: 24-25).

Por otro lado, la ELC, haciendo uso de las herramientas de LIP y de otras estrategias pedagógicas, busca avanzar en el acceso a la justicia y garantía de los derechos de las personas con escasos recursos económicos. La ELC llega a Colombia, impulsada por los movimientos del Derecho y Desarrollo, como una reacción al formalismo que caracterizaba los estudios jurídicos y la falta de consideración por las necesidades de las personas en situación de vulnerabilidad (Castro-Buitrago y otros, 2010: 78-80; Ramallo, 2020). En particular, la ELC se propone impulsar el aprendizaje del derecho a través de la práctica del mismo, al tiempo que impulsa la toma de responsabilidad de los estudiantes frente a la comunidad, enfatizando la responsabilidad social que implica la profesión legal y la reflexión ética sobre su práctica (Pelacani y Moreno, 2021: 227-230; Moreno, y Hoyos Ceballos, 2021). En este contexto, la Clínica Jurídica para Migrantes busca mejorar la situación de las personas migrantes y refugiadas y proteger sus derechos, más allá del caso estrictamente individual. Para esto utiliza el derecho como una herramienta de transformación social, consiente de las particularidades y retos específicos que implica atender esta población (Grimes, Honuskova y Stege, 2021).

En cuanto al litigio estructural, la Clínica trabaja en red con otros actores de la academia y de la sociedad civil con el propósito de incidir ante las autoridades y contribuir tanto al avance de la situación de los derechos de las personas migrantes y refugiadas en Colombia como al desarrollo de la política pública migratoria. Así, por ejemplo, implementamos estrategias para incidir en el escenario legislativo, en el marco del procedimiento de aprobación de la ley migratoria, al presentar un decálogo para indicar los mínimos de una ley migratoria con enfoque de derechos. ${ }^{3}$ También efectuamos intervenciones ante la Corte Constitucional y organismos internacionales al compartir información con la Comisión Interamericana de Derechos Humanos - en el marco de la visita in loco a Venezuela en junio del 2020-y la visita de trabajo a Colombia en junio del 2021.

Como complemento al trabajo de litigio, los estudiantes de la Clínica realizan brigadas jurídicas y Talleres de Empoderamiento Legal (TEL). Estos son una estrategia de incidencia enmarcada en la educación legal clínica (Grimes y otros, 2010: 225-240; Pandey y Shukkur, 2010: 241-252). A través de estos talleres se pretende que los estudiantes realicen jornadas de pedagogía de derechos para las personas migrantes y refugiadas con miras a que sean ellas quienes, de manera directa y sin la necesaria intervención de un abogado, puedan ejercer la defensa de sus derechos

3. Véase Dejusticia, «Colombia necesita una política migratoria con enfoque en derechos humanos», disponible en https://bit.ly/3ymydıb. 
mediante el uso de algunas estrategias legales a su alcance (MacDowell, 2008). En el contexto colombiano, mecanismos como la acción de tutela y el derecho de petición resultan fundamentales para avanzar los derechos de las personas en situación de vulnerabilidad y que experimentan obstáculos significativos para acceder a la justicia. Ello es así en la medida en que los instrumentos legales mencionados pueden ser interpuestos por cualquier persona, independiente de su origen nacional o estatus migratorio, y no requieren la representación de un profesional del derecho (Pelacani y Moreno, 2021).

\section{Los retos de atender a población migrante y refugiada en Colombia}

La educación legal clínica enfocada en la atención jurídica a personas migrantes y refugiadas conlleva retos específicos (Gordon, 2005; Ong Hing, 2008). Por un lado, por las características y necesidades particulares de esta población; por el otro, por las especificidades del contexto colombiano. El primer elemento que conviene resaltar es la poca atención que todavía recibe el derecho migratorio, y el estudio de la migración en general, en las facultades de Derecho de Colombia. Esto tiene como consecuencia que los estudiantes que llegan a ejercer su práctica jurídica en la Clínica carezcan de una formación mínima sobre esta área del derecho. Al mismo tiempo, la fragmentariedad y precariedad del marco normativo migratorio del Estado colombiano, su escasa previsibilidad y constante cambio añaden desafíos ulteriores para su conocimiento por parte de las y los jóvenes abogados (Moreno y Pelacani, 2020).

El segundo aspecto tiene que ver con la profunda vulnerabilidad de la población migrante y refugiada que se atiende. Por un lado, la situación migratoria irregular en la que suelen encontrarse la mayoría de los usuarios que acuden a la Clínica constituye el primer obstáculo al que se enfrentan los estudiantes en el diseño e implementación de estrategias para la protección de sus derechos. En diversas ocasiones, la falta de caminos posibles para que la persona regularice su situación migratoria reduce notablemente las herramientas legales para resolver el caso, mostrando a los estudiantes los límites del derecho.

Por otro lado, los escasos recursos económicos de los usuarios, en muchos casos pueden conllevar dificultades por la imposibilidad de acceder a medios de comunicación de forma constante o a una conexión a internet. De la misma manera, algunos usuarios presentan una alta movilidad al interior del país y en la región, lo cual también dificulta mantener la comunicación. Finalmente, se trata de personas que más allá de las necesidades legales, presentan también necesidades humanitarias insatisfechas, como el acceso a alimentación y a un alojamiento en condiciones dignas. Este es un aspecto que se ha manifestado de forma creciente, sobre todo en el contexto de la pandemia por covid-19. La imposibilidad de satisfacer en todos los casos las necesidades humanitarias de sus usuarios ha mostrado a los estudiantes, una vez más, las 
limitaciones del derecho y los ha llevado a reflexionar sobre los conflictos éticos que pueden surgir en la prestación de servicios legales a esta población, así como sobre los límites de su rol como abogados.

En la siguiente sección ilustramos dificultades a través de algunos de los asuntos más significativos que ha atendido la Clínica. Estos muestran como los aspectos mencionados anteriormente se relacionan y materializan.

\section{El acceso a la salud y al refugio}

Los casos de personas migrantes y refugiadas venezolanas con necesidades insatisfechas de atención en salud son los casos más numerosos recibidos por la Clínica desde su creación. El acceso a una atención integral en salud por parte de la población migrante y refugiada en Colombia depende, en primer lugar, de su situación migratoria (Amaya-Castro, Moreno y Pelacani, 2019). Sobre este derecho, además, se enfoca gran parte de la jurisprudencia de la Corte Constitucional colombiana sobre personas migrantes y refugiadas de nacionalidad venezolana (Moreno y Pelacani, 2020).

En la Clínica, los estudiantes se han enfrentado a casos en que los usuarios buscan acceder a un tratamiento de salud adecuado a la enfermedad que padecen, que se les rechaza o retrasa por su situación migratoria irregular y por no estar afiliados al sistema de seguridad social. Una negativa ante la cual se hace necesario acudir a la acción de tutela, con el fin de que el juez ordene la protección del derecho fundamental a la salud y a la vida digna de la persona migrante y, por ende, que se le practiquen los procedimientos y exámenes requeridos. Además, se trata de casos desarrollados durante la vigencia de las medidas para hacer frente a la pandemia por el covid-19. En este periodo, las dificultades de las personas migrantes y refugiadas para acceder a justicia y a los servicios de la Clínica se han visto incrementadas, al tiempo que su vulnerabilidad ha aumentado y así su necesidad de servicios legales.

Entre los casos más graves atendidos por la Clínica se destacan los de usuarios que han perdido la vida esperando recibir tratamiento, a pesar de contar con pronunciamientos favorables por parte de los jueces de tutela. Estos casos mostraron, nuevamente, la relación fundamental que existe entre el estatus migratorio de la persona migrante y refugiada y su acceso al derecho fundamental a la salud. En estos casos, el derecho mostró su ineficacia ante la falta de respuesta oportuna de las entidades ya que inclusive cuando el derecho de la persona había sido tutelado y su petición había recibido una respuesta favorable, esto no fue suficiente para que la persona recibiera atención oportuna y para preservar su vida.

Una situación similar se presenta respecto a las personas que solicitan el reconocimiento de la condición de refugiado, lo cual es, tal vez, uno de los aspectos más críticos en materia migratoria en el contexto colombiano. Según se indicó, la tramitación de solicitudes de refugio fue una de las primeras temáticas a las que se enfrentó 
la Clínica y tienen un peso muy significativo en la oferta de servicios legales que ofrece. Es así como a partir del litigio que adelantan los estudiantes se ha detectado que este procedimiento es poco garantista y respetuoso de las obligaciones internacionales adquiridas por Colombia (Moreno y Pelacani, 2021: 192-197; Mayorquín, 2019: 362-363).

A este respecto, una de las experiencias más fuertes que enfrentamos desde la Clínica fue la abrupta interrupción, debido a la emergencia por el covid-19, de los servicios a cargo de la autoridad migratoria, entre ellos, el cierre de la atención por parte de entidades públicas como Migración Colombia. Para las personas migrantes y refugiadas esta decisión fue devastadora porque les cerró, de facto, la interacción con la autoridad ante la cual debían adelantar trámites de forma permanente y que se hacían habitualmente de forma presencial.

Uno de estos trámites a cargo de Migración Colombia es, precisamente, la entrega de un salvoconducto que regulariza la situación migratoria de los solicitantes de refugio. Para ellos, en particular, esto implicó que no podían acudir a retirar sus salvoconductos o sus prórrogas. Los estudiantes de la Clínica experimentaron, de primera mano, cómo el sistema de seguridad social desvinculaba a nuestros usuarios toda vez que estos no podían demostrar su situación migratoria regular mediante el salvoconducto. En plena emergencia por el covid-19, uno de estos casos concluyó de manera fatal para uno de los usuarios de la Clínica que fue desvinculado del sistema de seguridad social, a pesar de su grave estado de salud y falleció prematuramente. ${ }^{4}$

Estos y otros casos similares conllevaron una carga emocional importante para los estudiantes que requirió atención y cuidado, así como la necesidad de una reflexión conjunta de más largo aliento sobre su práctica jurídica. Para atender las necesidades emocionales de los estudiantes fue fundamental dedicar algunas de las sesiones plenarias semanales de la Clínica a reflexionar sobre el fallecimiento de estas personas y la rabia e impotencia manifestada por las familias ante lo sucedido. También, en situaciones como estas, la universidad puso a disposición de los estudiantes canales para que recibieran el auxilio de profesionales en psicología de forma inmediata.

Además, al comienzo de la práctica jurídica en la Clínica los estudiantes reciben un taller sobre lo que se ha identificado como desgaste por empatía en personas que trabajan con poblaciones vulnerables. Este permite a las y los estudiantes conocer las causas que pueden llevar a este desgaste e identificar los comportamientos que les indican que pueden estar viviendo este tipo de experiencia. El taller comparte con los estudiantes herramientas para cuidarse en estas situaciones y los canales de atención a los que pueden acudir en caso de necesitarlo. Finalmente, la Clínica Jurídica es un curso donde la reflexión ética sobre la práctica legal constituye un elemento fundan-

4. Véase Milagros Palomares, «Mi papá murió de un cáncer esperando el papel», Semana, 20 de junio de 2020, disponible en https://bit.ly/3dKQ6in. 
te. Para ello constantemente se dedican espacios de reflexión sobre las implicaciones éticas de la profesión, con el fin de que los estudiantes compartan las dificultades y los conflictos éticos a los cuales se han enfrentado en el marco de la atención y los servicios que prestan a la población migrante y refugiada.

\section{El debido proceso y las sanciones migratorias}

Una de las experiencias más significativas que ha acumulado la Clínica en su tiempo de existencia tiene que ver con el derecho al debido proceso. En particular, se trata de casos en que personas migrantes y refugiadas fueron expuestas a procedimientos de expulsión y deportación con evidentes violaciones a su derecho fundamental al debido proceso. ${ }^{5}$ Además, en estos casos se ha comprometido el derecho a la unidad familiar de las personas migrantes sancionadas, así como el de sus hijos menores de edad, quienes son a su vez nacionales colombianos. ${ }^{6}$

La experiencia acumulada por la Clínica en esta materia nos ha mostrado que, en muchos casos, estas actuaciones son ultrasumarias y no se han agotado las distintas etapas del procedimiento previo a la sanción. Además, estos procedimientos expeditos concluyen en la adopción de decisiones administrativas carentes de motivación real y que no admiten ningún recurso. Otro aprendizaje importante en materia de debido proceso ha sido la constatación de las barreras de acceso a justicia que experimentan las familias que resultan separadas como consecuencia de la ejecución de las sanciones impuestas. Esto porque el hecho mismo del abandono del territorio por parte del extranjero sancionado le impide, de facto, conseguir asistencia legal y acceder a la justicia. Un aspecto común con el que hemos tenido que lidiar es, precisamente, perder contacto con las personas o con los miembros de sus familias. La escasez de información suficiente y de la documentación necesaria para la gestión y conocimiento a profundidad de los casos hace muy difícil el diseño y el adelantamiento de estrategias legales adecuadas y es un obstáculo que, incluso, nos obliga a desistir de los mismos.

Los asuntos que hemos adelantado en materia de debido proceso han tenido lugar, además, en momentos de gran convulsión social, como lo fue el Paro Nacional de 2019. En esa oportunidad, la población venezolana en Colombia fue señalada como responsable de actos de vandalismo y de la comisión de delitos, durante los días de protesta a lo largo del territorio colombiano, lo que llevó a la expulsión masiva de personas venezolanas con graves violaciones a su derecho fundamental al debido

5. Véase Laura Vásquez Roa, «La sospecha latente sobre los migrantes en Colombia», Rolling Stone. disponible en https://bit.ly/zjHtZNN.

6. Véase Carolina Moreno, «La unidad familiar de los migrantes: ¿De qué estamos hablando?», Revista Semana, 29 de noviembre de 2019, disponible en https://bit.ly/3xi2f69. 
proceso (Moreno, 2020). El acompañamiento de estos casos acarrea una gran exposición mediática, así como señalamiento y reproches por parte de autoridades y de distintos sectores de la sociedad que están de acuerdo con la expulsión expedita de las personas migrantes y refugiadas. Esto se debe a que, en estos casos, nuestros usuarios han sido acusados de "poner en riesgo la seguridad nacional». Acusaciones de esta naturaleza inundan de prejuicios los casos y generan percepciones adversas en las personas y los funcionarios. Como consecuencia se toman decisiones injustas y prematuras que son difíciles de encarar desde el derecho, entre otras razones, por la amplia discrecionalidad de la autoridad migratoria colombiana.

El litigio de estos casos, por las razones advertidas, es muy desafiante. Nos encontramos frente a situaciones que suceden con gran prontitud, lo que contrasta con la escasez de instrumentos legales para hacerles frente y con tiempos de espera prolongados que, en muchos casos, solo profundizan la vulnerabilidad que experimentan las personas expulsadas. El desgaste emocional por los retos que imponen los casos, el temor por los señalamientos y cuestionamientos, así como por la precariedad de las herramientas legales disponibles, son en todo caso una oportunidad para el aprendizaje y para ofrecer a los estudiantes una mirada crítica, aunque esperanzadora, del derecho. Los fracasos y la falta de respuestas oportunas o idóneas con las que nos confronta la práctica real del derecho en la Clínica pueden ser siempre un escenario de aprendizaje reflexivo y crítico que otros cursos tradicionales de la carrera tal vez no ofrecen.

\section{El potencial pedagógico y social transformador que subyace a la educación legal clínica}

Más allá de las particularidades de las temáticas antes descritas y del diseño de las estrategias de litigio que se ponen en marcha en cada caso, lo cierto es que en la experiencia de la Clínica Jurídica para Migrantes hemos combinado las estrategias de litigio individual y de litigio más estructural o de alto impacto (Ikawa, 2011: 197-210). A partir de los casos individuales que adelantamos es como mejor podemos conocer el marco normativo vigente, así como los vacíos de protección existentes. Estas evidencias son insumo clave para la construcción de las estrategias de orden estructural, conforme a las cuales buscamos una transformación social y legal más amplia (Alviar García, 2008).

Sin duda, los casos tratados por nuestros estudiantes de la Clínica les han permitido conocer de manera directa, y desde la experiencia, la extrema situación de vulnerabilidad de las personas que han tenido que dejar su país para preservar su vida y la de sus familias. Además, los practicantes han tenido la oportunidad de reconocer las injusticias que crea y promueve el derecho y constatar cómo el marco normativo puede profundizar y perpetuar la situación de vulnerabilidad de sus propios clientes 
y usuarios. El litigio de los casos es siempre una oportunidad para abordar críticamente el derecho, para humanizar el ejercicio de la profesión y para encontrar, en lo posible, una oportunidad para el uso del derecho como instrumento de cambio social (Wilson, 2017).

Así pues, el litigio de estos casos deja importantes aciertos desde el punto de vista pedagógico y de la educación legal clínica en particular. Por una parte, estos temas permiten a los miembros de la Clínica, tanto estudiantes como profesoras, reflexionar sobre las cuestiones problemáticas que presenta el marco normativo colombiano en materia migratoria, su fragmentariedad y sus vacíos de protección. En particular, los estudiantes han conocido de manera personal la excesiva discrecionalidad de la autoridad migratoria colombiana y el desconocimiento de algunos funcionarios y jueces sobre el contexto migratorio actual. Lo anterior, en muchas ocasiones, ha confrontado a los estudiantes con decisiones injustas, adversas o frente a las cuales el derecho no ofrece alternativas, todo lo cual es, en todo caso, una oportunidad significativa para el aprendizaje crítico y reflexivo. Estos casos han interpelado a los estudiantes sobre los límites del derecho, su falta de respuestas adecuadas desde un enfoque diferencial y de derechos, así como resultados tantas veces injustos. No obstante, su paso por la Clínica también ha permitido a los estudiantes recobrar alguna dosis de confianza en el derecho y, en particular, en el litigio de interés público como instrumento de transformación social.

Por último, los estudiantes practicantes también han tenido que lidiar con situaciones humanitarias complejas como las vivencias personales y familiares de sus usuarios, debido, entre otras razones, a su frágil situación socioeconómica. Por ejemplo, al comienzo de la pandemia por covid-19, nuestros practicantes se enfrentaron a situaciones como retorno no voluntario a Venezuela, hambre, deterioro de la salud, e incluso muerte y situación de calle. Escenarios frente a los cuales el derecho mostró ser insuficiente e incluso injusto. Frente a estas cuestiones críticas la Clínica, bajo la orientación permanente de las profesoras a cargo, propicia espacios de reflexión y diálogo que contribuyen a la formación de las y los futuros abogados. Reconocer estos obstáculos como parte del ejercicio del derecho, así como las responsabilidades sociales asociadas a la profesión legal, son todas piezas claves de la formación integral, ética y experta que la Clínica busca brindar a sus estudiantes practicantes.

\section{Conclusiones}

En este texto hemos querido reflexionar sobre cómo, desde la educación legal clínica, es posible responder a los desafíos de atender a personas migrantes y refugiadas, especialmente provenientes de Venezuela, en el contexto colombiano. En particular, ahondamos en la experiencia de la Clínica Jurídica para Migrantes, creada el 2019 en la Universidad de los Andes en Bogotá, para responder a las necesidades de servicios 
legales especializados por parte de esta población, en particular la que se encuentra en situación de vulnerabilidad. Para ello, reconstruimos los eventos que llevaron a la creación de la Clínica y reflexionamos sobre algunos asuntos que la misma ha acompañado. Esto con el fin de evidenciar los retos enfrentados por los estudiantes y las profesoras, así como los aprendizajes adquiridos.

La experiencia descrita en este texto, que se gestó en el Consultorio y que hoy continúa expandiéndose desde la Clínica, tiene un compromiso explícito de cambio social desde una enseñanza experiencial del derecho, enmarcada en la educación legal clínica o experiencial. Esta es una oportunidad valiosa para el aprendizaje desde la práctica, así como un vehículo para cambiar la realidad de las personas, en este caso, de las migrantes y refugiadas más empobrecidas que continúan llegando a Colombia.

$\mathrm{Al}$ margen de la cantidad de asuntos litigados y de personas migrantes y refugiadas atendidas, las profesoras de la Clínica tenemos un propósito explícito de impactar la formación legal de nuestros estudiantes mediante un proceso pedagógico que ocurre desde la experiencia. A su vez, desde ese aprendizaje experiencial, en la Clínica confiamos en las posibilidades de cambio social mediante el uso del derecho para la protección de los derechos de la población migrante y refugiada atendida. Esta apuesta por la transformación social, así sea en pequeñas dosis, merece toda la pena en una sociedad profundamente desigual como lo es la colombiana.

\section{Referencias}

ACNUR, Alto Comisionado de las Naciones Unidas para los Refugiados, y OIM, Organización Internacional para las Migraciones (2021). Plataforma de coordinación interagencial para refugiados y migrantes de Venezuela- $R_{4} V$. Disponible en https:// bit.ly/3hjmBqm.

Alviar García, Helena (2008). «The classroom and the clinic: The relationship between clinical legal education, economic development and social transformation». UCLA Journal of International Law and Foreign Affairs, 1 (13): 197-218.

Amaya-Castro, Juan Manuel, Carolina Moreno y Gracy Pelacani (2019). «La gestión de la migración en Colombia hoy: Propuesta de diálogo para una política pública con enfoque de derechos en educación y salud». Informes del Centro de Estudios en Migración (CEM). Informe 1-2019. Disponible en https://bit.ly/3hiRtHy.

BLOCH, Frank S. (2010). The global clinical movement: Educating lawyers for social justice. Oxford: Oxford University Press.

Bonilla, Daniel (coordinador) (2018). Abogados y justicia social: Derecho de interés público y clínicas jurídicas. Bogotá: Siglos del Hombre.

Castro-Buitrago, Erika, Nicolás Espejo-Yaksic, Mariela Puga y Marta Villarreal (2010). "Clinical legal education in Latin America: Toward public interest». En Frank S. Bloch (editor), The global clinical movement: Educating 
lawyers for social justice. Oxford: Oxford University Press. DOI: 10.1093/acprof:o so/9780195381146.003.0005.

Courtis, Christian (2007). «La educación clínica como práctica transformadora». En Marta Villarreal y Christian Courtis (editores), Enseñanza clínica del Derecho: Una alternativa a los métodos tradicionales de formación de abogados (pp. 9-24). Ciudad de México: ITAM.

García SuÁrez, Daniel Eduardo (2019). La formación de los profesionales socialmente responsables en la universidad: Una utopía posible en el currículo (pp. 88-100). Bogotá: Uniandes.

GonzÁlez Morales, Felipe (2004) «El trabajo público en materia de derechos humanos e interés público en América Latina». Cuadernos Deusto de Derechos Humanos, 27: 9-59. Disponible en https://bit.ly/3HNqWwZ.

GoRdon, Jennifer (2005). Suburban sweatshops: The fight for immigrant right. Cambridge: Belknap/Harvard University Press.

Grimes, Richard, David Mcquoid-Mason, Ed O’Brien y Judy Zimmer (2010). «Street law and social justice education». En Frank S. Bloch (editor), The global clinical movement: Educating lawyers for social justice (pp. 225-240). Oxford: Oxford University Press.

Grimes, Richard, Vera Honuskova y Ulrich Stege (2021). Teaching migration and asylum law: Theory and practice. Londres: Routledge.

IKAwA, Daniela (2011). «The Impact of Public Interest Law on Legal Education». En Frank S. Block (editor), The global clinical movement: Educating lawyers for social justice (pp. 197-210). Oxford: Oxford University Press. DOI: 10.1093/acprof:o so/9780195381146.003.0013.

MACDoweLL, Elizabeth L. (2008) «Law on the street: Legal narrative and the street law classroom». Rutgers Race \& the Law Review, 9 (2): 285-334.

MAYORQuín, Daniela (2019). «El refugio como alternativa a la migración forzada de venezolanos en Colombia». En Alexandra Castro Franco (editora), Venezuela Migra: Aspectos sensibles del éxodo hacia Colombia. Bogotá: Universidad Externado de Colombia.

Migración Colombia (2021). «Distribución de Venezolanos en Colombia: Corte 31 de enero de 2021». Disponible en https://bit.ly/3oUbTsJ.

Moreno, Carolina (2020). «El debido proceso legal: Un vacío de protección que experimentan los migrantes en Colombia». Border Criminologies. Disponible en https://bit.ly/3jIJoNL.

Moreno, Carolina y Gracy Pelacani (2020). «Corte Constitucional colombiana: $¿$ Un escenario posible para el experimentalismo constitucional en materia migratoria?». Latin American Law Review, 5: 139-157. DOI: https://doi.org/10.29263/ laro5.2020.07.

-. (2021). «El contexto de la migración venezolana en Colombia: Un análisis de la 
respuesta institucional en perspectiva regional». En Alejandro Fajardo y Alexandra Vargas (editores), Comunidad Venezuela: Una agenda de investigación y acción local (pp. 171-217). Bogotá: CODS-IDRC. Disponible en https://bit.ly/36eOQA4.

Moreno, Carolina y Esteban Hoyos Ceballos (2021). «La reflexión ética en la práctica jurídica de los estudiantes de derecho en Colombia: Navegar en la dicotomía entre justicia individual y justicia colectiva». En Sergio Iván Anzola Rodríguez, Juny Montoya Vargas, Isabel Cristina Jaramillo Sierra y Carlos Francisco Morales de Setién Ravina (editores), Ética profesional del abogado: Debates y tensiones (pp. 43-73). Bogotá: Uniandes.

ONG HING, Bill (2008). «Legal services support centers and rebellious advocacy: A case study of the immigrant legal resource center». Washington University Journal of Law and Policy, 28: 265-358.

Pandey, Ajay y Sheena Shukkur (2010) «Legal literacy projects: Clinical experience of empowering the poor in India». En Frank S. Bloch(editor), The global clinical movement: Educating lawyers for social justice (pp. 241-252). Oxford: Oxford University Press.

Pelacani, Gracy (2020). «Ley migratoria sí, pero no así». Universidad de los Andes. Disponible en https://bit.ly/3ymXrg3.

Pelacani, Gracy y Carolina Moreno (2021). «El fenómeno migratorio: La construcción de una "realidad más real" desde la educación experiencial». En Antonio Barreto y Eleonora Lozano (editores), Metodologías de investigación jurídica: Experiencias y desafíos del oficio de investigar en derecho (pp. 224-243). Bogotá: Uniandes.

Pelacani, Gracy, Carolina Moreno, Laura Dib-Ayesta y Mairene Tobón (2021). «Estatuto temporal de protección para migrantes venezolanos: Reflexiones de una política de regularización migratoria». Informes del Centro de Estudios en Migración (CEM). Informe 3-2021. Bogotá, Colombia. Disponible en https://bit. ly/3HPcZiw.

Proyecto Migración Venezuela (2020). «Vuelven los desalojos a migrantes: en julio ya irían más de mil». Disponible en https://bit.ly/37s9bCF.

Ramallo, María de los Ángeles (2020). «La tradición formalista en la enseñanza del derecho y su impacto en la formación práctica». Revista Pedagogía Universitaria y Didáctica del Derecho, 7 (1): 9-42. DOI: 10.5354/0719-5885.2020.54934.

ReCAlde, Gabriela, Tania Luna Blanco y Daniel Bonilla Maldonado (2017). «Justicia de pobres: Una genealogía de los consultorios jurídicos en Colombia». Revista de Derecho (Universidad del Norte), 47: 1-71. Disponible en https://bit.ly/3At4MMX. Wilson, Richard J. (2017). The global evolution of clinical legal education: More than a method. Cambridge: Cambridge University Press. 


\section{Sobre las autoras}

Gracy Pelacani es profesora asistente de la Facultad de Derecho de la Universidad de los Andes, Colombia, directora del Semillero de Investigación en Derecho y Migración en Colombia, cofundadora y profesora de la Clínica Jurídica para Migrantes, miembro del Centro de Estudios en Migración (CEM) y del Grupo de investigación Derecho, Migración y Acción Social (DMAS) de la Universidad de los Andes. Abogada de la Universitá degli Studi di Trento, Italia, y doctora en Estudios Jurídicos Comparados y Europeos de la misma universidad. Su correo electrónico es g.pelacani@ uniandes.edu.co. (D) https://orcid.org/oooo-0002-8801-8752.

Carolina Moreno es profesora asociada de la Facultad de Derecho de la Universidad de los Andes, Colombia, y directora de Investigaciones (Cijus), cofundadora de la Clínica Jurídica para Migrantes y directora del Centro de Estudios en Migración (CEM) y del Grupo de investigación Derecho, Migración y Acción Social (DMAS) de la misma universidad. Abogada y doctora en Derecho de la Universidad de los Andes y máster en Derecho Público de la Universidad Pompeu Fabra (Barcelona, España).Su correo electrónico es camoreno@uniandes.edu.co. (D) https://orcid. org/oooo-0001-6242-5029. 
La Revista Pedagogía Universitaria y Didáctica del Derecho (RPUDD) es una publicación científica semestral que contribuye a la reflexión multidisciplinaria sobre pedagogía universitaria y didáctica del derecho, para la formación y consolidación de esta área de investigación; así como a la difusión de prácticas innovadoras en la enseñanza-aprendizaje del derecho considerando el contexto nacional e internacional. Es una publicación electrónica internacional con una codirección entre Brasil y Chile.

\author{
DIRECTORA \\ María Francisca Elgueta Rosas \\ Universidad de Chile \\ DIRECTOR \\ Renato Duro Dias \\ Universidad Federal de Rio Grande, Brasil \\ SITIO WEB \\ pedagogiaderecho.uchile.cl \\ CORREO ELECTRÓNICO \\ rpedagogia@derecho.uchile.cl \\ LICENCIA DE ESTE ARTÍCULO
}

Creative Commons Atribución Compartir Igual 4.o Internacional

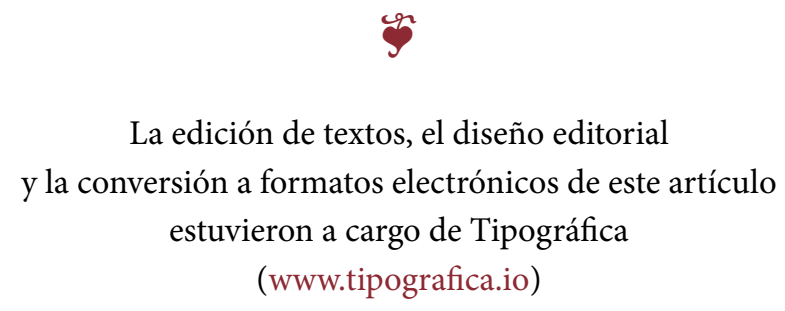

